\title{
Favorable Effects of Vitamin D Supplementation on Pregnancy Outcomes in Gestational Diabetes: A Double Blind Randomized Controlled Clinical Trial
}

\author{
Authors \\ Z. Asemi ${ }^{1}$, M. Karamali ${ }^{2}$, A. Esmaillzadeh ${ }^{3,4}$ \\ Affiliations \\ Affiliation addresses are listed at the end of the article
}

Key words

vitamin D supplementation

pregnancy outcomes

- GDM

pregnant women received 13.08.2014

accepted 07.10.2014

Bibliography

DOI http://dx.doi.org/

10.1055/s-0034-1394414

Published online:

November 5, 2014

Horm Metab Res 2015;

47: 565-570

(c) Georg Thieme Verlag KG

Stuttgart · New York

ISSN 0018-5043

\section{Correspondence}

\section{A. Esmaillzadeh, PhD}

Department of Community Nutrition

School of Nutrition and Food Science

Isfahan University of Medical

Sciences

PO Box 81745-151

Isfahan

I. R. Iran

Tel.: + 98/311/7922 720

Fax: +98/311/6681378

Esmaillzadeh@hlth.mui.ac.ir

\section{Abstract}

$\nabla$

Gestational diabetes mellitus (GDM) has been recognized as a significant risk factor for unfavorable pregnancy outcomes. Prevalence of vitamin D deficiency is highly prevalent among women with GDM. This study was designed to assess the effect of vitamin D supplementation on pregnancy outcomes of pregnant women with GDM who were not on oral hypoglycemic agents. This randomized controlled clinical trial was performed among 45 pregnant women diagnosed with GDM at 24-28 weeks' gestation. Subjects were randomly assigned to consume either vitamin D supplements (cholecalciferol) or placebo. Individuals in the vitamin $\mathrm{D}$ group $(\mathrm{n}=22)$ received $50000 \mathrm{IU}$ vitamin D3 pearl 2 times during the study: at study baseline and day 21 of intervention and those in placebo group $(n=23)$ received 2 placebos at the mentioned times. Fasting blood samples were taken

\begin{tabular}{ll}
\hline Abbreviations \\
25(OH)D & 25-Hydroxyvitamin D \\
CBR & Completed bed rest \\
CS & Cesarean section \\
FPG & Fasting plasma glucose \\
LGI & Low-glycemic index \\
GDM & Gestational diabetes mellitus \\
IUGR & Intrauterine growth retardation \\
LBW & Low birth weight \\
OGTT & Oral glucose tolerance test \\
PPROM & Premature preterm rupture of mem- \\
& brane
\end{tabular}

\section{Introduction}

$\nabla$

Gestational diabetes mellitus (GDM) is defined as glucose intolerance with onset or first recognition during pregnancy [1]. Prevalence of GDM at baseline to measure fasting plasma glucose. Participants underwent a 3-h oral glucose tolerance tests (OGTT) and the blood samples were collected at time 60,120 , and 180 min to measure plasma glucose levels. Newborn's weight, height, head circumference, Apgar score, and hyperbilirubinemia were determined. Taking vitamin D supplements, compared with placebo, resulted in improved pregnancy outcomes; such that those in the vitamin $\mathrm{D}$ group had no case of polyhydramnios, while $17.4 \%$ of subjects in placebo group had this condition $(\mathrm{p}=0.04)$. In addition, newborn's hyperbilirubinemia was significantly lower in vitamin $\mathrm{D}$ group than that in placebo group ( $27.3 \%$ vs. $60.9 \%, p=0.02)$. In conclusion, vitamin D supplementation for 6 weeks among pregnant women with GDM resulted in decreased maternal polyhydramnios and infant hyperbilirubinemia compared with placebo.

Clinical trial registration number www.irct.ir:IRCT201305115623N7.

during pregnancy ranges from 1-14\% of all pregnancies depending on the population studied and the diagnostic tests used [2]. The prevalence of this condition is $4.7 \%$ among Iranian pregnant women [3]. GDM is associated with short-term and long-term adverse health outcomes for both mothers and offspring [4]. Infants of diabetic mothers are at risk for physiologic, metabolic, and congenital complications such as preterm birth, macrosomia, asphyxia, respiratory distress, hypoglycemia, hypocalcemia, hyperbilirubinemia, polycythemia and hyperviscosity, hypertrophic cardiomyopathy as well as congenital anomalies, particularly of the central nervous system [5]. Women with GDM have higher rates of Cesarean section (CS) [6], are at increased risk of pregnancy-associated hypertension [7] and increased risk of perinatal morbidity and type 2 diabetes in later life $[5,8]$. 
Currently, no strong evidence exist regarding the best intervention for the management of GDM [9], however, a low-glycemic index diet (LGI), healthy diet according to recommendations for the general population [10] or an exercise program [11] could be beneficial. Accumulating evidence have reported a link between vitamin D deficiency and abnormal glucose metabolism. Epidemiological data have indicated that pregnant women with GDM were more likely to be vitamin D deficient [12]. In our previous study, we found improved metabolic profiles among pregnant women with GDM following the consumption of 100000 IU vitamin D supplements for 6 weeks [13]. Others have also reported the beneficial effects of vitamin $\mathrm{D}$ supplementation on metabolic status of GDM patients [14]. It is unclear if these improvements in metabolic profiles of GDM patients could be translated in improved pregnancy outcomes. Vitamin D supplementation in healthy nulliparous women did not influence pregnancy outcomes [15]. However, vitamin D supplementation might improve pregnancy outcomes of patients with GDM due to its effect on maternal insulin resistance, metabolic profiles [16], and increased metabolism of bile acids [17]. Therefore, we hypothesized that vitamin D supplementation might affect pregnancy outcomes of pregnant women with GDM. We are aware of no study examining the effect of vitamin D supplementation on pregnancy outcomes among women with GDM. The current study was, therefore, performed to investigate the effects of vitamin D supplementation on pregnancy outcomes in pregnant women with GDM who were not on oral hypoglycemic agents (OHAs).

\section{Subjects and Methods}

$\nabla$

Participants

This randomized double-blind placebo-controlled clinical trial was conducted at Kashan, Iran, during January 2013 to June 2013. For estimating sample size, we used a randomized clinical study sample size formula where type one $(\alpha)$ and type 2 errors ( $\beta$ ) were 0.05 and 0.20 (power $=80 \%$ ), respectively. Based on a previous study [18], we also considered 0.9 as SD and $0.55 \mathrm{~cm}$ as the difference in the mean (d) of newborns' length at birth as key variables. According to this, we needed 23 subjects in each group to have $80 \%$ power of the study. Pregnant women primigravida, aged $18-40$ years diagnosed with GDM by a $100 \mathrm{~g}$ oral glucose tolerance test at 24-28 weeks' gestation were recruited in this study. Participants in the study were not on OHAs. Gestational age was assessed from the date of last menstrual period and concurrent clinical assessment [19]. Pregnant women without a previous diagnosis of glucose intolerance were screened for GDM by 2 procedures. First, a $50 \mathrm{~g}$ glucose challenge test was used as preliminary screening. Individuals with 1 -h plasma glucose concentrations of $>140 \mathrm{mg} / \mathrm{dl}$ were then asked to participate in a $100 \mathrm{~g}$ oral glucose tolerance test (OGTT). Diagnosis of GDM was based on the criteria as set by the American Diabetes Association [20]: those whose plasma glucose met 2 of the following criteria were considered as having GDM: fasting $>95 \mathrm{mg} /$ $\mathrm{dl}, 1-\mathrm{h} \geq 180 \mathrm{mg} / \mathrm{dl}, 2-\mathrm{h} \geq 155 \mathrm{mg} / \mathrm{dl}$, and 3-h $\geq 140 \mathrm{mg} / \mathrm{dl}$. A total of 960 pregnant women attending maternity clinics affiliated to Kashan University of Medical Sciences, Kashan, Iran, were screened for GDM. Finally, 50 pregnant women met the inclusion criteria (900 women were excluded due to not having GDM and 10 women were excluded because of the diagnosis of GDM class A2 that needed insulin therapy: FPG $>105 \mathrm{mg} / \mathrm{dl}$ and BS 2-h postprandial $>120 \mathrm{mg} / \mathrm{dl}$ ). We excluded those with premature preterm rupture of membrane (PPROM), placenta abruption, pre-eclampsia, requiring to commence insulin therapy during intervention, hypothyroidism, urinary tract infection, smoking and kidney or liver diseases as well as those taking estrogen therapy. A total of 50 pregnant women were recruited in the study and after stratification for pre-intervention BMI $(<30$ and $\geq 30 \mathrm{~kg} / \mathrm{m}^{2}$ ) and weeks of gestation ( $<26$ or $\geq 26$ weeks), they were randomly assigned to consume vitamin D supplements $(n=25)$ or the placebo $(n=25)$ for 6 weeks. Random assignment was done by the use of computer-generated random numbers. A trained midwife at maternity clinic did the randomized allocation sequence and assigned participants to interventions. The study was double blind because patients and the investigators were not aware of the treatment any particular subject was receiving. The study was conducted according to the guidelines laid down in the Declaration of Helsinki. The ethical committee of Kashan University of Medical Sciences approved the study and informed written consent was obtained from all participants. The trial was registered in the Iranian website (www.irct.ir) for registration of clinical trials (IRCT code: IRCT201305115623N7).

\section{Study design}

At study baseline and after stratification for pre-intervention BMI and weeks of gestation, subjects were randomly assigned to receive either vitamin $D$ supplements (cholecalciferol) or placebo. Individuals in the vitamin D group received 50000 IU vitamin D3 pearl (D-Vitin 50000; Zahravi Pharm Co, Tabriz, Iran) 2 times during the study: at study baseline and day 21 of intervention and those in placebo group received 2 placebos (Barij Essence Co, Kashan, Iran) at the mentioned time. The duration of the study was 6 weeks. The appearance of the placebo capsules, their color, shape, size, and packaging were identical to the vitamin D3 capsules. Quality control of vitamin D supplements was done in the laboratory of Food and Drug Administration in Tehran, Iran by HPLC method. Following quality control, we found that the amount of cholecalciferol in the prescribed tablets was at the range of 47500-52500 IU. Participants were asked not to alter their routine physical activity or usual dietary intakes throughout the study and not to consume any supplements other than the one provided to them by the investigators. All subjects were also consuming $400 \mu \mathrm{g} / \mathrm{d}$ folic acid from the beginning of pregnancy and $60 \mathrm{mg} / \mathrm{d}$ ferrous sulfate from the second trimester. To assess compliance, patients were asked to bring the medication containers. Compliance was checked through counting unused pearls. To increase the compliance, all patients were receiving short messages on their cell phones to take the supplements each day. All pregnant women provided 3 dietary records ( 1 weekend day and 2 week days) and 3 physical activity records to make sure that they maintained their usual diet and physical activity during intervention. Both dietary and physical activity records were taken at weeks 2, 4, and 6 of intervention. Dietary intakes of participants throughout intervention were assessed by means of 3-day dietary records. The dietary records were based on estimated values in household measurements. To obtain nutrient intakes of participants based on these 3-day food diaries, we used Nutritionist IV software (First Databank, San Bruno, CA, USA) modified for Iranian foods. After diagnosis of GDM in patients attending the center, they were first instructed about the healthy diet; however, they were not given 
a specific menu and they were just participating in a nutritional education class that focused on basics of healthy diet.

\section{Assessment of anthropometric measures}

Anthropometric measurements were assessed at pre-pregnancy, baseline and after 6 weeks of intervention. Body weight was measured in an overnight fasting status without shoes in a minimal clothing state by the use of a digital scale (Seca, Hamburg, Germany) to the nearest $0.1 \mathrm{~kg}$. Height was measured using a nonstretched tape measure (Seca, Hamburg, Germany) to the nearest $0.1 \mathrm{~cm}$. Maternal weight was assessed at pre-pregnancy, baseline, and after 6 weeks of intervention in maternity clinics by trained midwifes. BMI was calculated as weight in $\mathrm{kg}$ divided by height in meters squared. Infants' length and weight were measured using standard methods (Seca 155 Scale, Hamburg, Germany) during the first $24 \mathrm{~h}$ after birth and were recorded to the nearest $1 \mathrm{~mm}$ and $10 \mathrm{~g}$, respectively. Infants' head circumference was measured to the nearest $1 \mathrm{~mm}$ with a Seca girth measuring tape. We also determined infants' 1- and 5-min Apgar score as another measure of pregnancy outcome. Macrosomic babies were defined as those whose birth weight was $>4000 \mathrm{~g}$ [21]. The ponderal index $\left(\mathrm{kg} / \mathrm{m}^{3}\right)$ of the infant was calculated.

\section{Biochemical and polyhydramnios assessment}

Fasting blood samples $(5 \mathrm{ml})$ were taken at baseline and after 6 weeks intervention at Kashan reference laboratory in an early morning after an overnight fasting. Blood samples were immediately centrifuged (Hettich, Tuttlingen, Germany) at $3500 \mathrm{rpm}$ for $10 \mathrm{~min}$ to separate serum. Then, the samples were stored at $-70^{\circ} \mathrm{C}$ before analysis at the KUMS reference laboratory. Plasma glucose levels that were measured on the day of blood sampling were quantified by the use of glucose oxidase/peroxidase (GOD-POD) method with commercially available kits (Pars Azmun Co, Tehran, Iran). Participants underwent a 3-h oral glucose tolerance tests (OGTT) and blood samples were collected at time 60,120 , and $180 \mathrm{~min}$ to measure plasma glucose levels. Serum 25-hydroxyvitamin D concentrations were assayed using a commercial ELISA kit (IDS, Boldon, UK). The inter- and intraassay CVs for serum 25-hydroxyvitamin D assays ranged from 5 to $7.5 \%$. Hyperbilirubinemia was considered when the total serum bilirubin level was at or above $15 \mathrm{mg} / \mathrm{dl}(257 \mathrm{~mol} / \mathrm{l})$ in infants $25-48 \mathrm{~h}$ old, $18 \mathrm{mg} / \mathrm{dl}(308 \mathrm{~mol} /)$ in infants $49-72 \mathrm{~h}$ old, and $20 \mathrm{mg} / \mathrm{dl}$ (342 mol/l) in infants older than $72 \mathrm{~h}$ [22]. Polyhydramnios was diagnosed with sonographic estimation method at post-intervention. On the basis of this measurement, polyhydramnios was defined as an amniotic fluid index (AFI) in excess of $25 \mathrm{~cm}$ [16].

\section{Statistical analysis}

To ensure the normal distribution of variables, Histogram and Kolmogorov-Smirnov test were applied. We used independent samples Student's $t$-test to detect differences in baseline measures as well as in dietary intakes between the 2 groups. Pearson Chi-square test was used for comparison of categorical variables. The effect of supplementation on outcome variables was examined through the use of repeated measure analysis of variance. In these analyses, treatment (vitamin D supplements vs. placebo) was considered as between-subject factor and time (Baseline vs. week 6 of intervention) as within-subject factor. A p-value of $<0.05$ was considered as statistically significant. All statistical analyses were done using Statistical Package for Social Sciences version 17 (SPSS Inc., Chicago, Illinois, USA).

\section{Results}

$\nabla$

Among individuals in the placebo group, 2 women [needed to commence insulin therapy $(n=1)$ or pre-eclampsia $(n=1)]$ were excluded. The exclusions in the vitamin D group were 3 persons [IUFD $(n=1)$, placenta abruption $(n=1)$, and CBR $(n=1)]$. Finally, 45 participants [placebo $(n=23)$ and vitamin $D(n=22)$ ] completed the trial ( $\odot$ Fig. 1). No side effects were reported following the consumption of vitamin D supplements in patients with GDM throughout the study.

Mean age of study participants was $30.9 \pm 5.8$ years. Gestational age, age, pre-pregnancy weight and BMI means was not statistically different between the 2 groups ( $\odot$ Table 1 ). Baseline weight and BMI as well as post-intervention means of these variables were not significantly different between vitamin $\mathrm{D}$ and placebo groups. Vitamin D supplementation resulted in a significant rise

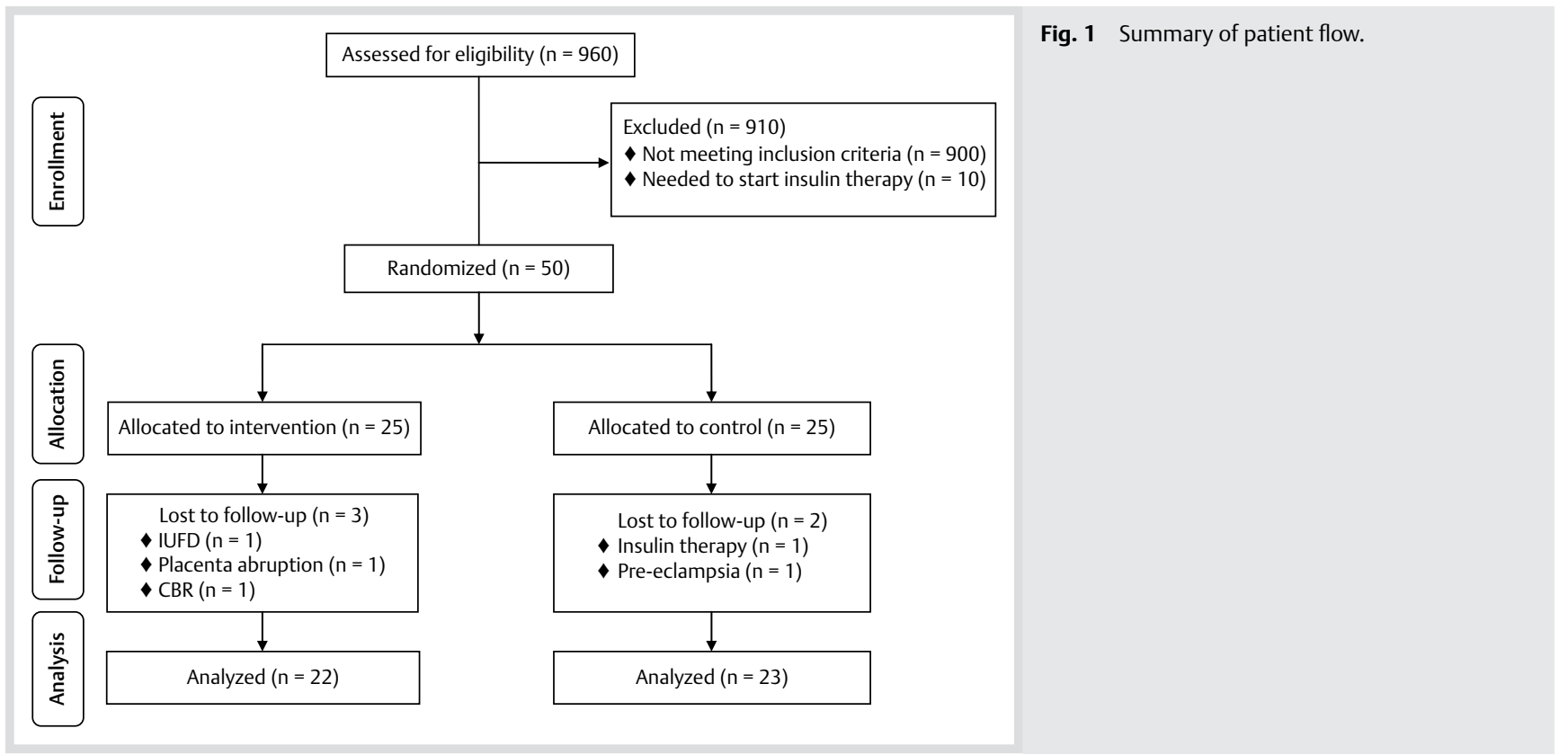




\begin{tabular}{|c|c|c|c|}
\hline & Placebo group $^{\dagger}(n=23)$ & Vitamin D group ${ }^{\dagger}(n=22)$ & $p^{\dagger i t}$ \\
\hline Maternal age (years) & $30.8 \pm 6.2$ & $31.1 \pm 5.5$ & 0.88 \\
\hline Height $(\mathrm{cm})$ & $159.6 \pm 4.5$ & $160.7 \pm 6.7$ & 0.53 \\
\hline Pre-pregnancy weight (kg) & $70.0 \pm 11.2$ & $70.2 \pm 9.0$ & 0.70 \\
\hline Weight at study baseline $(\mathrm{kg})^{*}$ & $77.8 \pm 12.9$ & $79.0 \pm 9.7$ & 0.73 \\
\hline Weight at end-of-trial (kg) & $79.5 \pm 12.5$ & $80.5 \pm 9.8$ & 0.77 \\
\hline Weight change $(\mathrm{kg})$ & $1.7 \pm 1.5$ & $1.5 \pm 0.6$ & 0.54 \\
\hline Pre-pregnancy BMI $\left(\mathrm{kg} / \mathrm{m}^{2}\right)^{*}$ & $27.1 \pm 4.0$ & $27.3 \pm 3.6$ & 0.87 \\
\hline BMI at study baseline $\left(\mathrm{kg} / \mathrm{m}^{2}\right)$ & $30.5 \pm 4.4$ & $30.7 \pm 3.9$ & 0.86 \\
\hline $\mathrm{BMI}$ at end-of-trial $\left(\mathrm{kg} / \mathrm{m}^{2}\right)$ & $31.1 \pm 4.3$ & $31.2 \pm 4.0$ & 0.92 \\
\hline BMI change $\left(\mathrm{kg} / \mathrm{m}^{2}\right)$ & $0.6 \pm 0.6$ & $0.5 \pm 0.2$ & 0.48 \\
\hline Vitamin D at study baseline (ng/ml) & $20.9 \pm 14.3$ & $18.9 \pm 14.5$ & 0.63 \\
\hline Vitamin D at end-of-trial (ng/ml) & $21.5 \pm 14.8$ & $40.4 \pm 27.0$ & 0.005 \\
\hline Vitamin D change $(\mathrm{ng} / \mathrm{ml})$ & $0.6 \pm 6.7$ & $21.5 \pm 21.4$ & $<0.0001$ \\
\hline Gestational age before intervention (weeks) & $25.8 \pm 1.3$ & $25.3 \pm 1.2$ & 0.17 \\
\hline \multicolumn{4}{|c|}{$\begin{array}{l}\text { Data are means } \pm \text { standard deviation } \\
\text { †Received placebo } 2 \text { times during the study: at study baseline and day } 21 \text { of intervention } \\
\text { ¡Received } 50000 \text { IU vitamin D } 3 \text { two times during the study: at study baseline and day } 21 \text { of intervention } \\
\text { ॠं Obtained from independent } t \text {-test }\end{array}$} \\
\hline
\end{tabular}

Table 1 General characteristics and serum vitamin $D$ levels of the study participants.

\begin{tabular}{|c|c|c|c|c|}
\hline & Placebo group ${ }^{\dagger}(n=23)$ & Vitamin D group ${ }^{\dagger \dagger}(n=22)$ & $p^{i t i}$ & \multirow{15}{*}{$\begin{array}{l}\text { Table } 2 \text { Dietary intakes of study } \\
\text { participants throughout the } \\
\text { study. }\end{array}$} \\
\hline Energy (kcal/d) & $2349 \pm 141$ & $2389 \pm 198$ & 0.42 & \\
\hline Fat $(g / d)$ & $83.4 \pm 11.5$ & $82.6 \pm 16.6$ & 0.84 & \\
\hline Protein $(\mathrm{g} / \mathrm{d})$ & $86.7 \pm 10.1$ & $85.9 \pm 17.9$ & 0.85 & \\
\hline Carbohydrate (g/d) & $320.3 \pm 32.3$ & $334.5 \pm 41.7$ & 0.21 & \\
\hline SFA $(g / d)$ & $24.4 \pm 5.6$ & $24.1 \pm 7.0$ & 0.85 & \\
\hline PUFA (g/d) & $27.4 \pm 5.9$ & $26.8 \pm 5.6$ & 0.74 & \\
\hline Cholesterol (mg/d) & $228.1 \pm 129.6$ & $190.5 \pm 127.5$ & 0.31 & \\
\hline Dietary fiber (g/d) & $17.5 \pm 3.5$ & $18.5 \pm 4.9$ & 0.39 & \\
\hline Selenium $(\mu \mathrm{g} / \mathrm{d})$ & $110.6 \pm 4.4$ & $117.7 \pm 3.7$ & 0.91 & \\
\hline Magnesium (mg/d) & $267.5 \pm 43.3$ & $286.1 \pm 73.6$ & 0.28 & \\
\hline Vitamin C (mg/d) & $182.3 \pm 92.3$ & $191.5 \pm 78.7$ & 0.72 & \\
\hline Calcium (mg/d) & $1137.3 \pm 171.9$ & $1150.9 \pm 187.5$ & 0.80 & \\
\hline Vitamin $D(\mu \mathrm{g} / \mathrm{d})$ & $2.9 \pm 0.9$ & $2.8 \pm 0.8$ & 0.91 & \\
\hline \multicolumn{4}{|c|}{$\begin{array}{l}\text { †Received placebo } 2 \text { times during the study: at study baseline and day } 21 \text { of intervention } \\
\text { †Received } 50000 \text { IU vitamin D3 two times during the study: at study baseline and day } 21 \text { of intervention } \\
\text { ॠं Obtained from independent } t \text {-test }\end{array}$} & \\
\hline
\end{tabular}

in serum 25-hydroxyvitamin D levels (21.5 vs. $0.6 \mathrm{ng} / \mathrm{ml}$, $\mathrm{p}<0.001$ ) compared with the placebo.

Based on 3-day dietary records obtained throughout the intervention, no statistically significant difference was seen between the 2 groups in terms of dietary intakes of energy, fats, proteins, carbohydrates, saturated fatty acids (SFA), polyunsaturated fatty acids (PUFA), cholesterol, dietary fiber, selenium, magnesium, vitamin C, calcium, and vitamin D ( $\odot$ Table 2 ).

Taking vitamin D supplements, compared with placebo, resulted in improved pregnancy outcomes; such that those in the vitamin D group had no case of polyhydramnios, while $17.4 \%$ of subjects in placebo group had this condition ( $0 \%$ vs. $17.4, p=0.04)$. In addition, newborn's hyperbilirubinemia was significantly lower in vitamin D group than that in placebo group ( $27.3 \%$ vs. $60.9 \%$, $\mathrm{p}=0.02$ ) ( $\odot$ Table 3 ). We did not find a significant difference in cesarean section rate, needing to insulin therapy after intervention, preterm delivery, newborn's birth size, ponderal index, and Apgar score comparing the 2 groups.

\section{Discussion $\nabla$}

The present study revealed that vitamin D supplementation for 6 weeks among pregnant women with GDM resulted in decreased maternal polyhydramnios and infant hyperbilirubinemia compared with placebo; however, we did not find any significant affect on cesarean section rate, needing to insulin therapy after intervention, newborn's birth size, and Apgar score. To the best of our knowledge, this is the first study reporting the effect of vitamin D supplementation on pregnancy outcomes of pregnant women with GDM.

Gestational diabetes is linked with several adverse pregnancy outcomes in maternal, offspring's, as well as later in their lives [8,23-25]. The current study showed that taking vitamin D supplements in pregnant women with GDM resulted in decreased polyhydramnios, but did not affect rate of cesarean section, need to commence insulin therapy after intervention and preterm delivery. In agreement with our study, vitamin D supplementation did not affect preterm delivery in healthy pregnant women [26]. Furthermore, vitamin D intake of $15-20 \mu \mathrm{g} / \mathrm{d}$, compared with less than $5 \mu \mathrm{g} / \mathrm{d}$, did not result in lower occurrence of preeclampsia among healthy nulliparous women [15]. Nonetheless, 


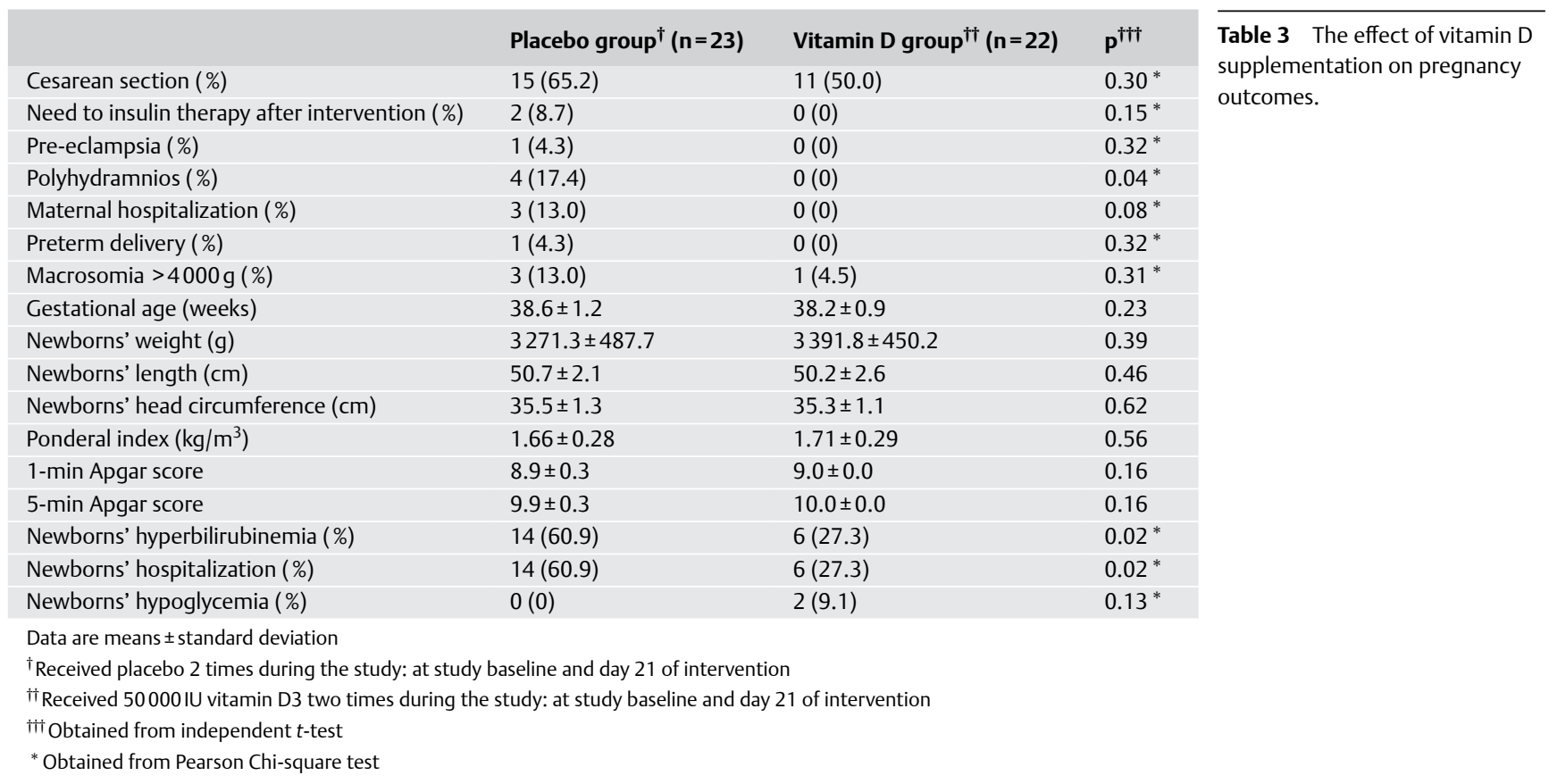

data on the effect of vitamin D supplementation on maternal, perinatal, or infant health outcomes in GDM patients are scarce. In a study by Koskinen et al. [27] lower 25(OH) D levels in amniotic fluid were found in diabetic mothers than normal pregnant women. The effect of supplementation on polyhydramnios in the current study might be attributed to its impact on decreased maternal insulin resistance [16]. Maternal hyperglycemia results in fetal hyperglycemia and fetal hyperinsulinemia, which in turn leads to enhanced glycogen synthesis, lipogenesis, increased protein synthesis, and thus fetal organomegaly and fat deposition. Our previous study showed that vitamin D supplementation for 6 weeks in GDM patients resulted in decreased serum insulin levels and insulin resistance [13].

We failed to find any significant effect of vitamin D supplementations on newborn's birth size, ponderal index and Apgar score. In line with ours, several observational studies did not show a relation between maternal vitamin $\mathrm{D}$ status and newborn's birth size. Mehta et al. [28] found no significant association between maternal vitamin D status among HIV-infected pregnant women and pregnancy outcomes including low birth weight and preterm birth. The same findings have also been reported in other investigations [29,30]. Ergocalciferol supplementation $(25 \mathrm{mg} / \mathrm{d})$ among pregnant women did not affect mean birth weight in other studies $[31,32]$. In opposite, taking either one oral dose of $1500 \mu \mathrm{g}$ vitamin D3 or 2 doses of $3000 \mu \mathrm{g}$ vitamin D3 in the second and third trimesters resulted in an increased birth weight, length, and head circumference [18]. Discrepancies between our findings and others might be related to different doses of vitamin D used, study designs and period of supplementation as well as subjects' characteristics.

We found that vitamin D supplementation in pregnant women with GDM resulted in decreased newborn's hyperbilirubinemia. Improved liver enzyme levels in cord blood (CB) of the infants whose mothers received one dose of $1500 \mu \mathrm{g}$ vitamin D3 in the second trimester or 2 doses of $3000 \mu \mathrm{g}$ vitamin D3 each in the second and third trimesters were seen compared with the controls [18]. In another study by Marya et al. [33] supplementation with 2 large doses of $600000 \mathrm{IU}$ each in the $7^{\text {th }}$ and $8^{\text {th }}$ months has led to a significant improvement in serum liver enzyme in maternal as well as cord sera. Several mechanisms can explain the effects of vitamin D supplementation on reduced newborn's hyperbilirubinemia. Active form of vitamin $D, 1 \alpha, 25-$ dihydroxyvitamin $\mathrm{D}_{3}$ induces vitamin $\mathrm{D}$ receptors (VDR), which in turn act as a receptor for secondary bile acids, such as lithocholic acid and 3-ketocholanic acid, and results in their catabolism via induction of cytochrome (CYP) 3 A enzymes [17,34]. In addition, maternal hyperglycemia and the subsequent induction of fetal hyperinsulinemia as well as reduced oxygenation are hypothesized to result in increased fetal oxygen uptake, fetal erythropoiesis, and subsequent hyperbilirubinemia [35].

Our study has some limitations. First is the duration of this trial. We were unable to continue the supplementation for more than 6 weeks due to the special condition of pregnant women. Second, we did not assess the effects of vitamin D supplementation on other pregnancy outcomes including neonatal respiratory distress syndrome and vitamin D concentrations in amniotic fluid.

In conclusion, vitamin D supplementation for 6 weeks among pregnant women with GDM resulted in decreased maternal polyhydramnios and hyperbilirubinemia compared with the placebo, but did not affect cesarean section rate, needing to insulin therapy after intervention, newborn's birth size, and Apgar score.

\section{Manuscript Contributions}

\section{$\nabla$}

Z.A. contributed in conception, design, statistical analysis and drafting of the manuscript. M.K. contributed in data collection and manuscript drafting. A.E. supervised the study. All authors read and approved the final version of the paper.

\section{Acknowledgements}

The present study was supported by a grant (no. 92151) from the Vice-Chancellor for Research, AUMS, Arak, Iran. 


\section{Conflict of Interest}

$\nabla$

None of the authors has any personal or financial conflict of interest.

\section{Affiliations}

Research Center for Biochemistry and Nutrition in Metabolic Diseases, Kashan University of Medical Sciences, Kashan, I. R. Iran

Department of Gynecology and Obstetrics, School of Medicine, Arak University of Medical Sciences, Arak, I. R. Iran

Food Security Research Center, Isfahan University of Medical Sciences,

Isfahan, I. R. Iran

${ }^{4}$ Department of Community Nutrition, School of Nutrition and Food Science, Isfahan University of Medical Sciences, Isfahan, I. R. Iran

\section{References}

1 Harlev A, Wiznitzer A. New insights on glucose pathophysiology in gestational diabetes and insulin resistance. Curr Diab Rep 2010; 10 : 242-247

2 Karcaaltincaba D, Kandemir O, Yalvac S, Guvendag-Guven S, Haberal A. Prevalence of gestational diabetes mellitus and gestational impaired glucose tolerance in pregnant women evaluated by National Diabetes Data Group and Carpenter and Coustan criteria. Int J Gynaecol Obstet 2009; 106: 246-249

3 Hossein-Nezhad A, Maghbooli Z, Vassigh AR, Larijani B. Prevalence of gestational diabetes mellitus and pregnancy outcomes in Iranian women. Taiwan J Obstet Gynecol 2007; 46: 236-241

4 Buchanan TA, Xiang AH, Page KA. Gestational diabetes mellitus: risks and management during and after pregnancy. Nat Rev Endocrinol 2012; 8: 639-649

5 Hay WW Jr. Care of the infant of the diabetic mother. Curr Diab Rep 2012; 12: 4-15

6 Reece EA. The fetal and maternal consequences of gestational diabetes mellitus. J Matern Fetal Neonatal Med 2010; 23: 199-203

7 Mastrogiannis DS, Spiliopoulos M, Mulla W, Homko CJ. Insulin resistance: the possible link between gestational diabetes mellitus and hypertensive disorders of pregnancy. Curr Diab Rep 2009; 9: 296-302

8 Linne $Y$, Barkeling B, Rossner S. Natural course of gestational diabetes mellitus: long term follow up of women in the SPAWN study. BJOG 2002; 109: 1227-1231

9 Oostdam N, van Poppel MN, Wouters MG, van Mechelen W. Interventions for preventing gestational diabetes mellitus: a systematic review and meta-analysis. J Womens Health (Larchmt) 2011; 20: 1551-1563

10 Louie JC, Brand-Miller JC, Markovic TP, Ross GP, Moses RG. Glycemic index and pregnancy: a systematic literature review. J Nutr Metab 2010; 2010: 282464

11 Jelsma JG, van Poppel MN, Galjaard S, Desoye G, Corcoy R, Devlieger $R$, van Assche A, Timmerman D, Jans G, Harreiter J, Kautzky-Willer A, Damm P, Mathiesen ER, Jensen DM, Andersen L, Dunne F, Lapolla A, Di Cianni G, Bertolotto A, Wender-Oegowska E, Zawiejska A, Blumska K, Hill D, Rebollo P, Snoek FJ, Simmons D. DALI: Vitamin D and lifestyle intervention for gestational diabetes mellitus (GDM) prevention: an European multicentre, randomised trial - study protocol. BMC Pregnancy Childbirth 2013; 13: 142

12 Alzaim $M$, Wood RJ. Vitamin D and gestational diabetes mellitus. Nutr Rev 2013; 71: 158-167

13 Asemi Z, Hashemi T, Karamali M, Samimi M, Esmaillzadeh A. Effects of vitamin D supplementation on glucose metabolism, lipid concentrations, inflammation, and oxidative stress in gestational diabetes: a double-blind randomized controlled clinical trial. Am J Clin Nutr 2013; 98: 1425-1432

14 Rudnicki PM, Molsted-Pedersen L. Effect of 1,25-dihydroxycholecalciferol on glucose metabolism in gestational diabetes mellitus. Diabetologia 1997; 40: 40-44
15 Haugen M, Brantsaeter AL, Trogstad L, Alexander J, Roth C, Magnus P, Meltzer HM. Vitamin D supplementation and reduced risk of preeclampsia in nulliparous women. Epidemiology 2009; 20: 720-726

16 Nobile de Santis MS, Radaelli T, Taricco E, Bertini S, Cetin I. Excess of amniotic fluid: pathophysiology, correlated diseases and clinical management. Acta Biomed 2004; 75 (Suppl 1): 53-55

17 Makishima M, Lu TT, Xie W, Whitfield GK, Domoto H, Evans RM, Haussler $M R$, Mangelsdorf DJ. Vitamin D receptor as an intestinal bile acid sensor. Science 2002; 296: 1313-1316

18 Kalra P, Das V, Agarwal A, Kumar M, Ramesh V, Bhatia E, Gupta S, Singh $S$, Saxena $P$, Bhatia $V$. Effect of vitamin D supplementation during pregnancy on neonatal mineral homeostasis and anthropometry of the newborn and infant. Br J Nutr 2012; 108: 1052-1058

19 Gupta $P$, Narang M, Banerjee BD, Basu S. Oxidative stress in term small for gestational age neonates born to undernourished mothers: a case control study. BMC Pediatr 2004; 4: 14

20 Rossi G. Diagnosis and classification of diabetes mellitus. Recenti Prog Med 2010; 101: 274-276

21 Boulet SL, Alexander GR, Salihu HM, Pass M. Macrosomic births in the united states: determinants, outcomes, and proposed grades of risk. Am J Obstet Gynecol 2003; 188: 1372-1378

22 Porter ML, Dennis BL. Hyperbilirubinemia in the term newborn. Am Fam Physician 2002; 65: 599-606

23 Bener A, Saleh NM, Al-Hamaq A. Prevalence of gestational diabetes and associated maternal and neonatal complications in a fast-developing community: global comparisons. Int J Womens Health 2011; 3: $367-373$

24 Gabbe SG, Graves CR. Management of diabetes mellitus complicating pregnancy. Obstet Gynecol 2003; 102: 857-868

25 Jelsema RD. Management of diabetes mellitus complicating pregnancy. Obstet Gynecol 2004; 103: 586 author's reply 587

26 Thorne-Lyman A, Fawzi WW. Vitamin D during pregnancy and maternal, neonatal and infant health outcomes: a systematic review and meta-analysis. Paediatr Perinat Epidemiol 2012; 26 (Suppl 1): 75-90

27 Koskinen T, Kuoppala T, Tuimala R. Amniotic fluid 25-hydroxyvitamin $\mathrm{D}$ concentrations in normal and complicated pregnancy. Eur J Obstet Gynecol Reprod Biol 1986; 21: 1-5

28 Mehta S, Hunter DJ, Mugusi FM, Spiegelman D, Manji KP, Giovannucci EL, Hertzmark E, Msamanga GI, Fawzi WW. Perinatal outcomes, including mother-to-child transmission of HIV, and child mortality and their association with maternal vitamin D status in Tanzania. J Infect Dis 2009; 200: 1022-1030

29 Biesalski HK. Vitamin E requirements in parenteral nutrition. Gastroenterology 2009; 137: S92-S104

30 Morley R, Carlin JB, Pasco JA, Wark JD. Maternal 25-hydroxyvitamin $\mathrm{D}$ and parathyroid hormone concentrations and offspring birth size. J Clin Endocrinol Metab 2006; 91: 906-912

31 Delvin EE, Salle BL, Glorieux FH, Adeleine P, David LS. Vitamin D supplementation during pregnancy: effect on neonatal calcium homeostasis. J Pediatr 1986; 109: 328-334

32 Mallet E, Gugi B, Brunelle P, Henocq A, Basuyau JP, Lemeur H. Vitamin D supplementation in pregnancy: a controlled trial of two methods. Obstet Gynecol 1986; 68: 300-304

33 Marya RK, Rathee $S$, Lata V, Mudgil S. Effects of vitamin D supplementation in pregnancy. Gynecol Obstet Invest 1981; 12: 155-161

34 Xie W, Radominska-Pandya A, Shi Y, Simon CM, Nelson MC, Ong ES Waxman DJ, Evans RM. An essential role for nuclear receptors SXR/ PXR in detoxification of cholestatic bile acids. Proc Natl Acad Sci USA 2001; 98: 3375-3380

35 Ferrara A, Weiss NS, Hedderson MM, Quesenberry CP Jr, Selby JV, Ergas IJ, Peng T, Escobar GJ, Pettitt DJ, Sacks DA. Pregnancy plasma glucose levels exceeding the American Diabetes Association thresholds, but below the National Diabetes Data Group thresholds for gestational diabetes mellitus, are related to the risk of neonatal macrosomia, hypoglycaemia and hyperbilirubinaemia. Diabetologia 2007; 50: 298-306

Note of Concern
Since publication of this article, serious concerns have been
raised about the integrity of the reported methods, results
and analysis. Responses by the leading author and ethics
committees have been unsatisfactory and inconclusive; we
advise readers to interpret the information presented in the
article with due caution.

